\title{
Low-dose Btk inhibitors: an 'aspirin' of tomorrow?
}

\author{
Bernard Payrastre ${ }^{1,2}$ and Agnès Ribes ${ }^{1,2}$ \\ ${ }^{\prime}$ INSERM U1048 and Université Toulouse III Paul Sabatier and '²aboratoire d'Hématologie, CHU de Toulouse, Toulouse Cedex 03, France. \\ E-mail: BERNARD PAYRASTRE - bernard.payrastre@inserm.fr
}

doi:10.3324/haematol.2020.265173

B ruton tyrosine kinase (Btk) is one of the five members of the Tec family of cytoplasmic non-receptor protein tyrosine kinases. Expressed in hematopoietic cells, with the exception of T cells, it is essential for B-cell maturation and signal transduction of the B-cell antigen receptor. Loss-of-function mutations in its gene lead to an immunodeficiency disease called X-linked agammaglobulinemia. Since the 2000s, Btk has emerged as a major therapeutic target in the treatment of B-cell malignancies including chronic lymphocytic leukemia, mantle-cell lymphoma and Waldenström macroglobulinemia. ${ }^{1}$ Ibrutinib is the first-in-class irreversible inhibitor of Btk acting by covalent modification of the cysteine residue C418 in the kinase ATP binding domain. In the long-term, daily intake of this drug has remarkable activity on these malignant blood disorders. ${ }^{1}$ There are, however, some side effects, including skin rashes, atrial fibrillation, hypertension, and increased risk of bleeding, in about $40 \%$ of treated patients. ${ }^{2}$ While most bleeding events are of low grade, some grade 3 or higher events are reported in $5 \%$ of patients. $^{2}$ Interestingly, in this issue of Haematologica, Nicolson and colleagues propose a therapeutic application for Btk inhibitors at low doses in thrombosis. ${ }^{3}$

Two members of the Tec family expressed in platelets, Btk and Tec, are involved in signal transduction downstream of platelet receptors containing an immunoreceptor tyrosinebased activation motif (ITAM) or a closely related hemITAM. ${ }^{4}$ Glycoprotein VI (GPVI), a platelet-specific collagen receptor constitutively associated with the ITAM-containing FcR $\gamma$ chain, uses Btk to phosphorylate phospholipase C $\gamma 2$ (PLC $\gamma 2){ }^{5}$ PLC $\gamma 2$-mediated calcium mobilization and protein kinase $C$ activation are critical for collagen-evoked platelet activation and aggregation. ${ }^{4}$ Tec can partially compensate for the absence of Btk downstream of GPVI. ${ }^{6}$ The hemITAM-containing C-type lectin-like receptor 2 (CLEC-2), a podoplanin receptor highly expressed in platelets, also uses Btk and Tec., ${ }^{4,7}$ Finally, the platelet-specific receptor for von Willebrand factor, GPIb, which does not contain either ITAM or hemITAM, activates Btk. ${ }^{8}$

Following the observation of an increased bleeding risk in patients treated with ibrutinib, several studies have demonstrated that the drug affects platelet functions in vitro and $e x$ vivo through both on- and off-target effects., ${ }^{2,-12}$ Consistent with the fact that patients with X-linked agammaglobulinemia do not experience increased bleeding, ibrutinib-related hemorrhagic syndrome in patients with B-cell malignancies would be mainly due to off-target effects of the drug. ${ }^{12-14}$ Ibrutinib has, for instance, been shown to affect Src-family kinases (SFK) in platelets. ${ }^{12-14}$ The relatively high doses of ibrutinib used in the treatment of chronic lymphocytic leukemia (420 mg/day) and mantle-cell lymphoma (560 mg/day) likely contribute to the platelet effect via inhibition of Btk, Tec and also off-targets such as SFK. Second-generation Btk inhibitors with greater specificity for Btk, including acalabrutinib and tirabrutinib, ${ }^{1}$ have less impact on platelets and, compared to ibrutinib, are associated with a lower risk of severe bleeding in treated patients. ${ }^{2,12-14}$ These drugs do not abolish the mild bleeding diathesis that may be influenced by intrinsic features of B-cell malignancies in addition to the anti-platelet effect of the drugs. ${ }^{2,12-14}$

Nicolson and colleagues report that low doses of Btk inhibitors selectively block platelet activation dependent on CLEC-2. ${ }^{3}$ Such low doses only delay platelet aggregation induced by GPVI triggering. The authors propose that the differential effect of Btk inhibition in CLEC-2, relative to GPVI signaling, is due to a positive feedback loop involving thromboxane A2 and adenosine diphosphate. This important Btkdependent positive feedback control from thromboxane A2 and adenosine diphosphate receptors observed in human platelets stimulated through CLEC-2 was not found in mouse platelets. Accordingly, mouse platelets require higher concentrations of ibrutinib to block CLEC-2-mediated activation. Furthermore, contrasting with the conclusion of a previous study, the authors convincingly demonstrated that the Syk tyrosine kinase lies upstream of Btk in the CLEC-2 signaling cascade. The mandatory role of Btk downstream of CLEC-2 was confirmed by using platelets from patients with X-linked agammaglobulinemia, which were unable to aggregate and phosphorylate PLC $\gamma 2$ on the Btk phosphorylation site in response to CLEC-2 activation. Interestingly, Tec was not able to compensate for the lack of Btk in the CLEC-2 signaling. Nicolson et al. ${ }^{3}$ also showed that while Btk is important through both its kinase activity and its docking protein properties downstream of GPVI, only its kinase activity is critical for platelet activation by CLEC-2.

As an application of their observation, Nicolson and colleagues ${ }^{3}$ proposed that low doses of ibrutinib could prevent deep vein thrombosis (DVT), as platelet CLEC-2 was previously shown to play a key role in this pathology. ${ }^{15}$ This hypothesis was tested in an experimental mouse model of DVT, known to be dependent on platelet CLEC-2. In the group of 13 mice treated with ibrutinib $(35-70 \mathrm{mg} / \mathrm{kg})$ a trend towards a reduction of the prevalence of inferior vena cava thrombus was observed compared to that in the control group. The authors suggest that the lack of statistical significance of this reduction may have resulted from an incomplete inhibition of CLEC-2 signaling through Btk pharmacological blockade within the 48 hours required for venous thrombosis in this experimental model, and from the fact that mouse platelet CLEC-2 is less critically dependent on Btk than is human platelet CLEC-2. Among the small number of mice that formed a thrombus despite ibrutinib treatment, the thrombus size was comparable to that in the control group, suggesting that CLEC- 2 could primarily act in the phase of thrombus initiation.

Considering the critical role of CLEC-2 in DVT ${ }^{15}$ as well as in thrombo-inflammation induced by bacterial infection, ${ }^{16}$ and its weak contribution to normal hemostasis, ${ }^{4}$ these results suggest that a low concentration of Btk inhibitors could be an 


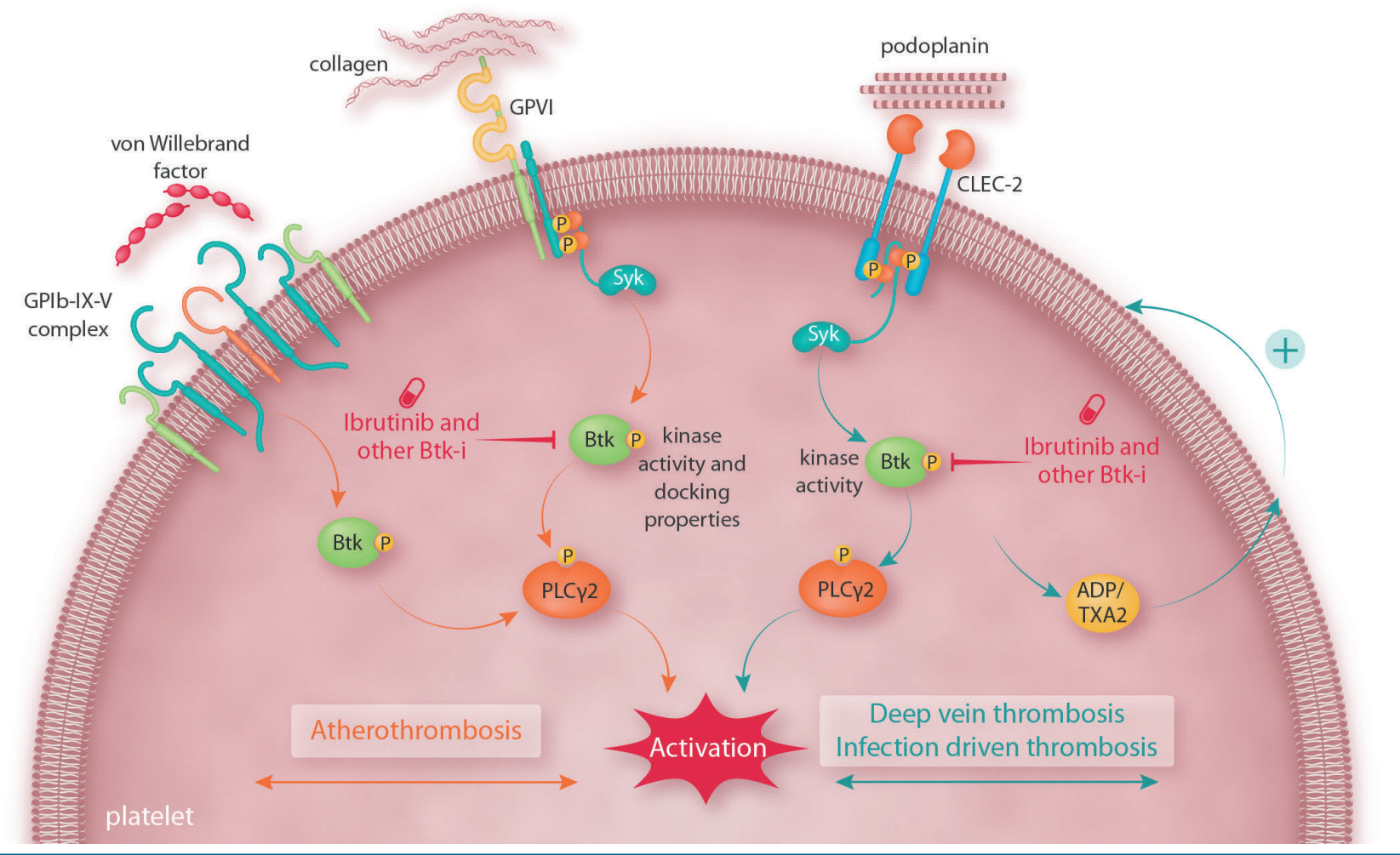

Figure 1. Btk inhibition downstream of platelet GPVI and CLEC-2 as a new strategy to prevent deep vein thrombosis, thrombo-inflammation and atherothrombosis This schematic and simplified representation highlights the role of Btk in the intracellular signaling pathways evoked by platelet receptors such as GPVI, GPIb-IX-V and CLEC-2. Low doses of Btk inhibitors, compared to the relatively high doses currently used for the treatment of B-cell malignancies, strongly prevent CLEC-2evoked platelet activation and may protect against deep vein thrombosis and infection-driven thrombosis. The Btk-dependent positive feedback loop involving adenosine diphosphate and thromboxane A2 in human platelets stimulated by CLEC-2 triggering is shown. Moreover, inhibiting Btk downstream of GPVI (strongly triggered by plaque collagen), and to a lesser extent GPIb, may protect against atherothrombosis. Low-dose irreversible inhibitors may be more effective on platelets that lack de novo synthesis of Btk than on nucleated cells such as B cells. It will, however, be important to determine the appropriate dose of Btk inhibitors to use, as some individuals are more sensitive than others. ${ }^{14}$ GP: glycoprotein; CLEC-2: C-type lectin-like receptor; Btk-i: Bruton tyrosine kinase inhibitor; Btk: Bruton tyrosine kinase; PLC 2 2: phospholipase C $\gamma 2$; ADP: adenosine diphosphate; TXA: thromboxane A2.

option to prevent thrombotic complications in these situations.

The study by Nicolson and colleagues ${ }^{3}$ is the second example showing that low-dose Btk inhibitors could be considered as potential antithrombotic drugs. Recently, it was reported that low doses of Btk inhibitors, including ibrutinib and the novel irreversible inhibitors, prevent platelet thrombus formation, at arterial blood flow rates, on human atherosclerotic plaques obtained during carotid endarterectomy. ${ }^{17}$ The authors suggest that this atherosclerotic plaque-selective platelet activation was blocked due to the targeting of signal transduction of GPVI (strongly activated by plaque collagen), and to a lesser extent GPIb, by Btk inhibitors. Optimization of GPVI signaling inhibition by Btk inhibitors led to the demonstration that low blood concentrations of inhibitors are sufficient for GPVI-selective platelet inhibition relevant for atherothrombosis, while sparing primary hemostasis. ${ }^{18}$ This is consistent with the fact that, like CLEC-2, GPVI has a minimal role in normal hemostasis. ${ }^{4}$ Through a small pilot study, the authors could show that low-dose ibrutinib (140 mg/day or every 2 days for a week) was sufficient to prevent atherosclerotic plaque-induced platelet thrombus formation under flow ex vivo and was more effective than aspirin (100 mg/day). ${ }^{17-19}$

Whether CLEC-2-mediated Btk activation is also targeted in the prevention of atherothrombosis by Btk inhibitors was not investigated in these studies. However, since podoplanin (which is not expressed in the normal vascular wall) has been found in atheromatous plaque, it is tempting to speculate that blockade of the CLEC-2/Btk axis also contributed to this effect. ${ }^{20}$ This could explain the pronounced efficacy of Btk inhibitors at relatively low doses in this condition. Finally, there is a recent report that ibrutinib treatment could reduce platelet-mediated inflammation, contributing to atherogenesis in an obese Rhesus macaque model of early atherosclerosis. ${ }^{21}$

In conclusion, Nicolson and colleagues bring important new information on the contribution of Btk in the platelet CLEC-2 signaling cascade and propose that targeting Btk downstream of CLEC-2 could protect against DVT and thrombo-inflammation. ${ }^{3}$ This study, alongside those from Busygina and colleagues ${ }^{17-19}$ and Kohs and colleagues, ${ }^{21}$ shed light on the potential application of Btk inhibitors in vascular and cardiovascular diseases (Figure 1). Considering the very active development of Btk inhibitors in cancer treatment, such tyrosine kinase inhibition-based antiplatelet therapy, rather than more conventional approaches targeting platelet-specific surface receptors, is attractive. One can, however, question whether the exciting results summarized above are a sufficient proof-ofconcept to open up a new therapeutic orientation for Btk inhibitors. The fact that the GPVI/Btk and CLEC-2/Btk axes are weakly involved in normal hemostasis and more 
strongly implicated in thrombotic complications such as DVT, thrombo-inflammation and atherothrombosis is a powerful argument. However, several issues remain to be resolved before undertaking clinical trials to evaluate the efficacy of low doses of Btk inhibitors compared to current antithrombotic treatments in these pathologies. For example, do we need to inhibit both Btk and Tec to obtain an antithrombotic effect in the different situations? Do low doses of Btk inhibitors preferentially inhibit Btk in platelets and spare B-cell functions? Do they prevent thrombus formation in vivo in other relevant animal models of DVT, thrombo-inflammation and atherothrombosis, such as rabbits, and do they spare homeostasis of the lymphatic system?

It would also be useful to tackle more clinical issues, such as: (i) the potential relative protection of patients with X-linked agammaglobulinemia and oncology patients under Btk inhibitors against DVT and atherothrombotic complications; (ii) the impact of low doses of Btk inhibitors on the side effects observed with long-term treatment with high doses of ibrutinib, such as atrial fibrillation; or (iii) the potential efficacy of reversible Btk inhibitors in preventing thrombotic events with shortterm applications. Let us bet that future studies will resolve these issues as such a new treatment is of obvious appeal in the prevention of thrombosis in different pathological situations.

\section{Disclosures}

No Conflicts of interest to disclose.

\section{Contributions}

$B P$ and $A R$ wrote the manuscript and made the figure.

\section{Acknowledgments}

Thanks are due to our colleagues from INSERM-U1048 (I2MC) and the Hematology Laboratory of the CHU-Toulouse, France.

\section{References}

1. Singh SP, Dammeijer F, Hendriks R. Role of Bruton's tyrosine kinase in B cells and malignancies. Mol Cancer. 2018;17(1):57.

2. Shatzel JJ, Olson SR, Tao DL, McCarty OJT, Danilov AV, Deloughery TG. Ibrutinib-associated bleeding: pathogenesis, management and risk reduction strategies. J Thromb Haemost. 2017;15(5):835-847.

3. Nicolson PLR, Nock SH, Hinds J, et al. Low-dose Btk inhibitors selec- tively block platelet activation by CLEC-2. Haematologica. 2021;106(1):208-219

4. Rayes J, Watson SP, Nieswandt B. Functional significance of the platelet immune receptors GPVI and CLEC-2. J Clin Invest. 2019;129(1):12-23.

5. Quek LS, Bolen J, Watson SP. A role for Bruton's tyrosine kinase (Btk) in platelet activation by collagen. Cur Biol. 1998;8(20):1137-1140.

6. Atkinson BT, Ellmeier W, Watson SP. Tec regulates platelet activation by GPVI in the absence of Btk. Blood. 2003;102(10):3592-3599.

7. Manne BK, Badolia R, Dangelmaier C, et al. Distinct pathways regulate Syk protein activation downstream of immune tyrosine activation motif (ITAM) and hemITAM receptors in platelets. J Biol Chem. 2015;290(18):11557-11568

8. Liu J, Fitzgerald ME, Berndt MC, Jackson CW, Gartner TK. Bruton tyrosine kinase is essential for botrocetin/VWF-induced signaling and GPIbdependent thrombus formation in vivo. Blood. 2006;108 (8):2596-2603.

9. Levade M, David E, Garcia C, et al. Ibrutinib treatment affects collagen and von Willebrand factor-dependent platelet functions. Blood. 2014;124(26):3991-3995.

10. Bye AP, Unsworth AJ, Vaiyapuri S, Stainer AR, Fry MJ, Gibbins JM Ibrutinib inhibits platelet integrin $\alpha \operatorname{Ilb} \beta 3$ outside-in signaling and thrombus stability but not adhesion to collagen. Arterioscler Thromb Vasc Biol. 2015;35(11):2326-2335.

11. Kamel S, Horton L, Ysebaert L, et al. Ibrutinib inhibits collagen-mediated but not ADP-mediated platelet aggregation. Leukemia. 2015;29(4):783-787.

12. Nicolson PLR, Hughes CE, Watson S, et al. Inhibition of Btk-specific concentrations of ibrutinib and acalabrutinib delays but does not block platelet aggregation to GPVI. Haematologica. 2018;103(12): 2097-2108.

13. Bye AP, Unsworth AJ, Desborough MJ, et al. Severe platelet dysfunction in NHL patients receiving ibrutinib is absent in patients receiving acalabrutinib. Blood Adv. 2017;1(26):2610-2623.

14. Series J, Garcia C, Levade M, Viaud J, Sié P, Ysebaert L, Payrastre B. Differences and similarities in the effect of ibrutinib and acalabrutinib on platelet functions. Haematologica. 2019;104(11):2292-2299.

15. Payne H, Ponomaryov T, Watson SP, Brill A. Mice with a deficiency in CLEC-2 are protected against deep vein thrombosis. Blood. 2017;129(14):2013-2020.

16. Hitchcock JR, Cook CN, Bobat S, et al. Inflammation drives thrombosis after salmonella infection via CLEC-2 on platelets. J Clin Invest. 2015;125(12):4429-4446.

17. Busygina K, Jamasbi J, Seiler T, et al. Oral Bruton tyrosine kinase inhibitors selectively block atherosclerotic plaque-triggered thrombus formation in humans. Blood. 2018;131(24):2605-2616.

18. Denzinger V, Busygina K, Jamasbi J, et al. Optimizing platelet GPVI inhibition versus haemostatic impairment by the Btk inhibitors ibrutinib, acalabrutinib, ONO/GS-4059, BGB-3111 and evobrutinib. Thromb Haemost. 2019;119(3):397-406.

19. Busygina K, Denzinger V, Bernlochner I, Weber C, Lorenz R, Siess W. Btk inhibitors as first oral atherothrombosis-selective antiplatelet drugs? Thromb Haemost. 2019:119(8):1212-1221.

20. Inoue O, Hokamura K, Shirai T, Vascular smooth muscle cells stimulate platelets and facilitate thrombus formation through platelet CLEC-2: implications in atherothrombosis. PLoS One. 2015;10(9): e0139357.

21. Kohs $\mathrm{T}$, Olson $\mathrm{S}$, Xie A et al. Effect of BTK inhibition on plateletmediated inflammation in an obese Rhesus macaque model of early atherosclerosis. ISTH 2020 Congress abstract. Res Pract Thromb Haemost 2020;4 (Suppl 1):PB0044. 